\title{
APPLICATION OF CENTRAL COMPOSITE DESIGN TO OPTIMIZE THE AMOUNT OF CARBON SOURCE AND PREBIOTICS FOR BIFIDOBACTERIUM BIFIDUM BB01
}

\author{
Guowei SHU*1, Ni LEI*, He CHEN*, Man HU*, Hui YANG* \\ *School of Food and Biological Engineering, Shaanxi University of Science \\ \& Technology, Xi'an 710021 China
}

\begin{abstract}
The objective of the present study was to obtain the optimum proportion of the carbon source and prebiotics for Bifidobacterium bifidum BBO1 by the central composite design (CCD). The effect of carbon source (lactose) and two prebiotics (inulin and fructooligosaccharides) on the $\mathrm{BB} 01$ were observed by measuring the $\mathrm{OD}_{600}$ value, $\mathrm{pH}$ value and the viable counts at $18 \mathrm{~h}$. The final optimized concentrations of carbon source and prebiotics were: lactose $1.6 \%$, inulin $0.26 \%$, and fructooligosaccharides $0.22 \%$. The result indicates that the growth of $B$. bifidum BBO1 shows an significant increase in the optimized culture medium $(\mathrm{p}<0.05)$, the $\mathrm{OD}_{600}$ value reached 1.434 at $18 \mathrm{~h}$, which increased $6.58 \%$ compared to the control. And the viable counts of B. bifidum BBO1 increased $24.36 \%$ and reached $(2.17 \pm 0.06) \times 10^{9} \mathrm{cfu} / \mathrm{mL}$. The results show that the optimization of the carbon source and prebiotics using CCD in this study is workable and necessary.
\end{abstract}

Keywords: Bifidobacterium bifidum, central composite design, prebiotics, probiotics

\section{INTRODUCTION}

Probiotics are micro-organisms selected mostly from bacteria that form a part of the normal intestinal microflora of humans. They are live microorganisms which are beneficial to the host when ingested in sufficient amounts (Wang et al.,2012). Probiotics contained by yogurts are claimed to provide several benefits to people such as enhance lactose utilisation (De Vrese et al., 2001), inhibit cancer (Rafter, 2004), keep the balance of intestinal microflora

\footnotetext{
${ }^{1}$ Corresponding author. Mailing address: Guowei Shu, 37\# School of Food and Biological Engineering, Shaanxi University of Science \& Technology, Weiyang district, Xi'an city, Shaanxi Province, China. E-mail:shuguowei@gmail.com
} 
(Mainville et al., 2005) and decrease serum cholesterol level (Baroutkoub et al., 2010).

Bifidobacteria are increasingly recognized as potential probiotics with advantageous properties; they are important species of the intestinal tract and associated with a healthy status in humans (Holzapfel et al., 1998). In addition, yogurt containing Bifidobacterium bifidum could improve the production of immunoglobulin A (IgA) in the intestine, which could improve the local immunity of the host against intestinal disease (Kabeerdoss et al., 2011). Also, it could inhibit some food borne pathogens (Goderska and Czarnecki, 2007) and decrease gastrointestinal infections due to the producing of the antimicrobial substances such as organic acids, deconjugated bile acids, hydrogen peroxide, antibiotics and bacteriocins (Schiffrin and Blum, 2001).

Single factor experiment or orthogonal experiment could only optimize one or several factors at a time, which may cause the missing of some potential influential factors involved (Kalil et al., 2000). The factorial design and response surface methodology (RSM) had been used in many study fields, which would well reveal and predict the interaction of the different components in the medium (Veronique et al., 1983; Hendrix, 1980). It could save the time and labor and can also find out the optimum levels of the variation. Different levels for one factor depends different experimental design. RSM mainly include the box-behnken design (BBD), the central composite design (CCD), the D-optimal design, the user-defined design, and the historical data design. Among then, The CCD and BBD were relatively popular designs for RSM, which had 3 and 5 levels respectively for one numeral factor (Box and Wilson, 1951). The growth of B. bifidum had the relatively high demand for the culture medium to meet, sole carbon source was hard to meet the growth need of B. bifidum, therefore, it is necessary to add the compound carbon source and prebiotics to optimize the medium, which would promote the growth of the strain, shorten the growth circle and increase economic benefit.

In our previous study, the single factor test and the Plackett-Burman design were employed to choose the main influence factors. According to the results of them, the steepest ascent experiment was also conducted; the carbon source (lactose) and prebiotics (inulin and fructooligosaccharides) were selected to do the further experiment. The objective of the present study was to apply the central composite design to optimize the carbon source and prebiotics for $B$. bifidum $\mathrm{BB} 01$ based on the previous work. 


\section{MATERIALS AND METHODS}

Strain and culture medium: The bacterium used in this work was Bifidobacterium bifidum BB01 (BB01), which was provided by School of Food and Biological Engineering, Shaanxi University of Science and Technology.

B. bifidum $\mathrm{BB} 01$ was grown and activated in MRS (lactose) culture medium containing $20 \mathrm{~g}$ lactose, $10 \mathrm{~g}$ peptone, $4 \mathrm{~g}$ yeast extract, $8 \mathrm{~g}$ beef extract, $2 \mathrm{~g}$ Dibasic Ammonium Citrate, $2 \mathrm{~g} \mathrm{~K}_{2} \mathrm{HPO}_{4}$, $3 \mathrm{~g}$ sodium acetate, $0.2 \mathrm{~g} \mathrm{MgSO}_{4}$, $0.05 \mathrm{~g} \mathrm{MnSO}_{4}, 0.5 \mathrm{~g}$ L-cysteine hydrochloride, $1 \mathrm{~mL}$ Tween 80 , and then were dissolved in $1000 \mathrm{~mL}$ distilled water with an initial $\mathrm{pH}$ of $6.2-6.4$, the culture was sterilized at $118 \square$ for $15 \mathrm{~min}$.

MRS agar medium (Hope Bio-technology CO. LTD, Qingdao, China) sterilized at $121 \square$ for 15 min, which was used as Counting Culture Medium.

Activation and incubation of strain: $5 \%$ inoculum size of $B$. bifidum BBOI was added in the MRS (lactose) culture medium and incubated at $37^{\circ} \mathrm{C}$ for $24 \mathrm{~h}$, using microscope to ensure there were no mixed bacterium in the medium, then added 5\% inoculum size of B. bifidum BBO1 to the MRS (lactose) culture medium to activate the strain for three times, after activation, incubated the $\mathrm{B}$. bifidum $\mathrm{BB} 01$ at $37^{\circ} \mathrm{C}$ for $18 \mathrm{~h}$. The transfers of strain were operated in the super-clean worktable.

Determination of growth curve: $5 \%$ inoculum size of B. bifidum BBO1 was added in the MRS (lactose) culture medium and incubated at $37^{\circ} \mathrm{C}$ for $24 \mathrm{~h}$, measuring the $\mathrm{OD}$ value at $600 \mathrm{~nm}$, the $\mathrm{pH}$ value and the viable counts of BB01 every $2 \mathrm{~h}$, the growth curve was drawn on the basis of these three responses.

Experimental design: The effect of carbon source (glucose, lactose, maltose, sucrose) and prebiotics (fructooligosaccharides, xylo-oligosaccharide, oligoisomaltose, galacto-oligosaccharides, stachyose, inulin) on the growth of BB01 was studied by single factor test and Plackett-Burman (PB) design. The results showed that lactose, glucose, fructooligosaccharides, xylooligosaccharide, galacto-oligosaccharides and inulin had positive effect on the growth of BB01. Lactose, inulin and fructooligosaccharides were selected to conduct the steepest ascent experiment. And according to the result of steepest ascent, the center point was regard as level 0 of significant factors. Central composite design (CCD) and response surface methodology were employed to do the further experiment, and finally make certain the amount of the carbon source and prebiotics in culture medium. The CCD and RSM were carried out using SAS software (Version, 9.1.3).

Determination of OD value and $\mathbf{p H}$ value: Determing the optical density value (OD) at $600 \mathrm{~nm}$ with a spectrophotometer (SP-756PC, Shanghai 
Spectrum Co., Ltd., Shanghai, China). The $\mathrm{pH}$ value of the culture media was measured by a $\mathrm{pH}$ meter (pHS-3C Shanghai Precision Scientific Instrument Co., Ltd, Shanghai, China).

Evaluation of the viable counts of $B$. bifidum BB01: Gradient dilution method was employed to measure the viable counts of BB01, added $1 \mathrm{~mL}$ diluted bacterial suspension to $9 \mathrm{~mL}$ sterile saline solution, make serial dilution through this method, remove $1 \mathrm{~mL}$ dilution bacterial suspension to the MRS high layered medium, cultured at $37^{\circ} \mathrm{C}$ for $48 \mathrm{~h}$, then chose the number of bacteria that was in the range of 30-300, two dilution was needed for each sample, and three copies were conducted to work out the mean value for each dilution. The unit of viable counts was $\mathrm{cfu} / \mathrm{mL}$.

Statistic analyses: The effect of different levels of factors on the B. bifidum $B B 01$ were statistically analyzed by means of variance analysis (ANOVA) and response surface methodology with SAS (Version, 9.1.3) software. The response surface plot and contour plots were also drawn using SAS.

\section{RESULTS AND DISCUSSIONS}

Optimization of the compound carbon source and prebiotics: According to the significant factors and levels, the compound carbon source and prebiotics of the culture medium was further optimized using a three-factor, five-level central composite design (CCD) in this study, each group conducted three parallel experiments. The coded values of different levels of each variable are shown in Table 1.

Table 1. The factors level coding table of Central Composite Design

\begin{tabular}{cccc}
\hline Level & X1 Lactose $(\%)$ & X2 Inulin(\%) & $\begin{array}{c}\text { X3 Fructo } \\
\text { oligosaccharides(\%) }\end{array}$ \\
\hline-1.682 & 1.432 & 0.206 & 0.206 \\
-1 & 1.5 & 0.220 & 0.220 \\
0 & 1.6 & 0.240 & 0.240 \\
1 & 1.7 & 0.260 & 0.260 \\
1.682 & 1.768 & 0.274 & 0.274 \\
\hline
\end{tabular}

The experimental design and the result of response value $\mathrm{Y} 1$ ( the viable counts of BB01 in the culture medium) are given in Table 2. According to the experimental result from Table2, using SAS software to build response surface model and analyze the data. the regression equation of this experiment is: 
$\mathrm{Y} 1=1.771615-0.036741 \times \mathrm{X} 1+0.041161 \times \mathrm{X} 2-0.081903 \times \mathrm{X} 3-0.199505 \times \mathrm{X} 1 \times \mathrm{X} 1-0.027 \times$ $\mathrm{X} 1 \times \mathrm{X} 2+0.002 \times \mathrm{X} 1 \times \mathrm{X} 3-0.166271 \times \mathrm{X} 2 \times \mathrm{X} 2-0.322 \times \mathrm{X} 2 \times \mathrm{X} 3-0.167155 \times \mathrm{X} 3 \times \mathrm{X} 3$ In this equation, $\mathrm{Y} 1$ means the predicted value of viable counts of Bifidobacterium bifidum in the cultural medium. X1, X2, X3 represent the coded values of lactose, inulin, and fructooligosaccharides respectively.

Table 2. The experimental design and results of Central Composite Design

\begin{tabular}{|c|c|c|c|c|}
\hline RUN & $\mathrm{X} 1$ & $\mathrm{X} 2$ & $\mathrm{X} 3$ & $\begin{array}{c}\mathrm{Y} 1 \quad\left(\times 10^{9} \mathrm{cfu} / \mathrm{mL}\right. \\
)\end{array}$ \\
\hline 1 & -1 & -1 & -1 & 0.997 \\
\hline 2 & -1 & -1 & 1 & 1.485 \\
\hline 3 & -1 & 1 & -1 & 1.713 \\
\hline 4 & -1 & 1 & 1 & 0.910 \\
\hline 5 & 1 & -1 & -1 & 1.000 \\
\hline 6 & 1 & -1 & 1 & 1.493 \\
\hline 7 & 1 & 1 & -1 & 1.605 \\
\hline 8 & 1 & 1 & 1 & 0.813 \\
\hline 9 & -1.68179 & 0 & 0 & 1.280 \\
\hline 10 & 1.68179 & 0 & 0 & 1.097 \\
\hline 11 & 0 & -1.68179 & 0 & 1.135 \\
\hline 12 & 0 & 1.68179 & 0 & 1.430 \\
\hline 13 & 0 & 0 & -1.68179 & 1.430 \\
\hline 14 & 0 & 0 & 1.68179 & 1.130 \\
\hline 15 & 0 & 0 & 0 & 1.733 \\
\hline 16 & 0 & 0 & 0 & 1.740 \\
\hline 17 & 0 & 0 & 0 & 1.773 \\
\hline 18 & 0 & 0 & 0 & 1.787 \\
\hline 19 & 0 & 0 & 0 & 1.750 \\
\hline 20 & 0 & 0 & 0 & 1.723 \\
\hline 21 & 0 & 0 & 0 & 1.800 \\
\hline 22 & 0 & 0 & 0 & 1.813 \\
\hline 23 & 0 & 0 & 0 & 1.832 \\
\hline
\end{tabular}


Regression equation obtained was evaluated by analysis of variance (ANOVA) and tested the significant level. The results were shown in Table 3.

Table 3. The ANOVA of Central Composite Design

\begin{tabular}{|c|c|c|c|c|c|c|}
\hline SOURCE & DF & $\mathrm{SS}$ & MS & $\mathrm{F}$ & $p$ & Sig. \\
\hline $\mathrm{X} 1$ & 1 & 0.018 & 0.018 & 6.034 & 0.0289 & $*$ \\
\hline $\mathrm{X} 2$ & 1 & 0.023 & 0.023 & 7.573 & 0.0165 & $*$ \\
\hline X3 & 1 & 0.092 & 0.092 & 29.983 & 0.000106 & $* * *$ \\
\hline $\mathrm{X} 1 * \mathrm{X} 1$ & 1 & 0.632 & 0.632 & 206.985 & 0.0001 & $* * *$ \\
\hline $\mathrm{X} 1 * \mathrm{X} 2$ & 1 & 0.006 & 0.006 & 1.909 & 0.1904 & \\
\hline $\mathrm{X} 1 * \mathrm{X} 3$ & 1 & $3.2 \times 10^{-5}$ & $3.2 \times 10^{-5}$ & 0.010 & 0.9201 & \\
\hline $\mathrm{X} 2 * \mathrm{X} 2$ & 1 & 0.439 & 0.439 & 143.768 & 0.0001 & $* * *$ \\
\hline $\mathrm{X} 2 * \mathrm{X} 3$ & 1 & 0.829 & 0.829 & 271.473 & 0.0001 & $* * *$ \\
\hline $\mathrm{X} 3 * \mathrm{X} 3$ & 1 & 0.444 & 0.444 & 145.301 & 0.0001 & $* * *$ \\
\hline Model & 9 & 2.464 & 0.274 & 89.594 & 0.0001 & $* * *$ \\
\hline Linear & 3 & 0.133 & 0.044 & 14.530 & 0.0002 & $* * *$ \\
\hline Square & 3 & 1.495 & 0.498 & 163.122 & 0.0001 & $* * *$ \\
\hline Cross Product & 3 & 0.835 & 0.278 & 91.131 & 0.0001 & $* * *$ \\
\hline Residual error & 13 & 0.040 & 0.003 & & & \\
\hline Lack of fit & 5 & 0.028 & 0.006 & 3.823 & 0.0457 & $*$ \\
\hline Pure error & 8 & 0.012 & 0.001 & & & \\
\hline Total & 22 & 2.503 & & & & \\
\hline
\end{tabular}

From the ANOVA of Table 3, we can see the P-value of regression model was 0.0001 , which means the model was extremely significant and the analysis of data was in effect. In addition, the regression coefficient $\mathrm{R}^{2}$ value of $98.41 \%$ and $\mathrm{Adj}^{2}$ value of $97.31 \%$ indicates the fit of regression equation was acceptable. The F-value was 89.594 (F > F0.01), which shows the description of linear relation between response value and factors with this equation is significant. And this method is workable. In one degree term of this regression equation, the $\mathrm{X} 2$ and $\mathrm{X} 3$ are significant, while all of the squares are significant. This suggests the relation between response value and the factors are not simple linear relation. The F-value of the correlation 
$\mathrm{X} 2 * \mathrm{X} 3$ is relatively big, which means there are some interaction between them.

Variation tendency of the response value (Y1) caused by factors are given in Fig. 1 , we can see clearly that $\mathrm{X} 1 * \mathrm{X} 1, \mathrm{X} 2 * \mathrm{X} 2, \mathrm{X} 3 * \mathrm{X} 3$ are all extremely significant $(\mathrm{p}<0.001)$, the curvature effects of three factors has significant influence on the viable counts of Bifidobacterium Bifidium (Y1), extremum all appears at inflexion point in the three graphs. Among then, Effect of X1 on $\mathrm{Y} 1$ increased as the increasing of $\mathrm{X} 1$, after peaking at center point, $\mathrm{Y} 1$ gradually decreased as the increasing of X1. Effect of X2, X3 on Y1 has the same tendency with X1.

95X Prediction Intervals

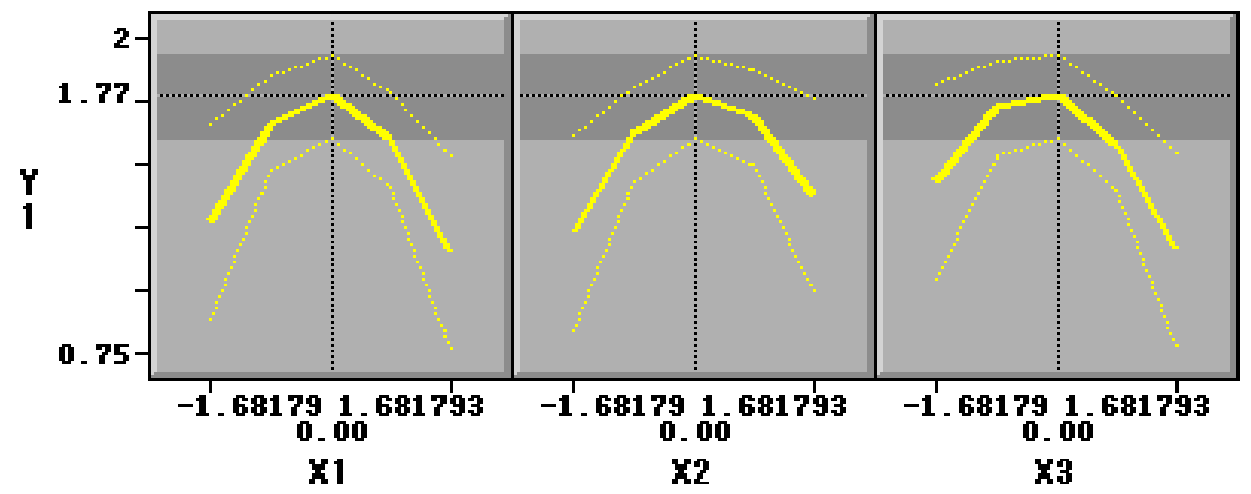

Figure 1. The trends of response value $\mathrm{Y}$ with factors

Interactions among the variables on the viable counts of $B$. bifidum BBO1 Using software to analyze the regression equation,the response surface plot and contour plot obtained by equation were shown in Figures 2, 3 and 4.
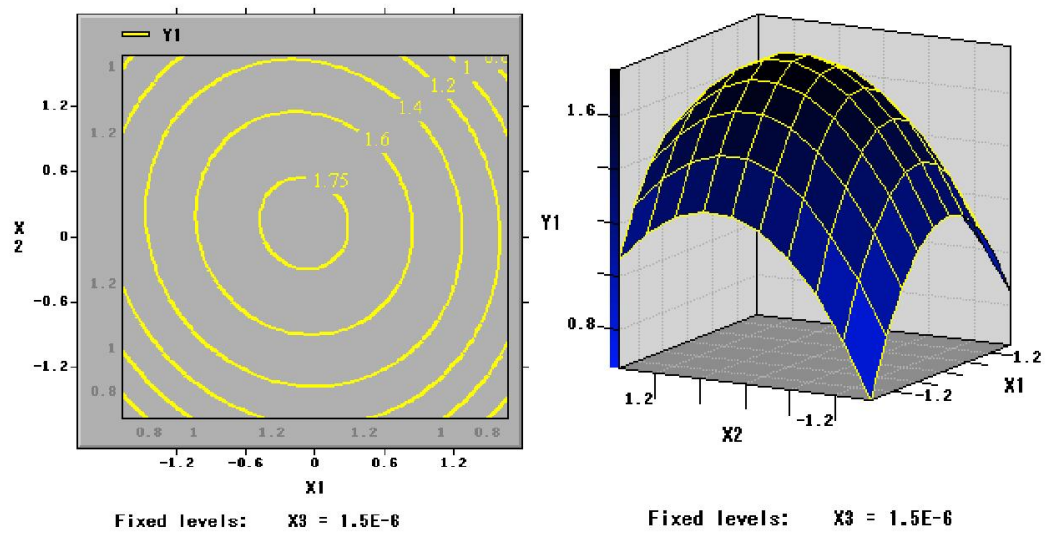

Figure 2. Contour plot and Response surface plot of lactose and inulin on the viable counts of BB01 


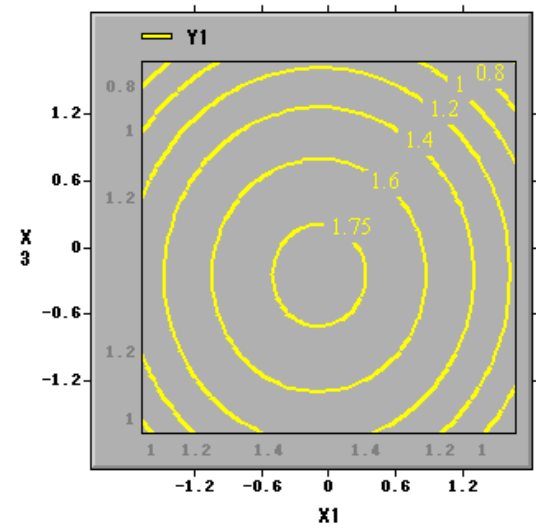

Fixed levels: $\quad \mathrm{X} 2=1.5 \mathrm{E}-6$

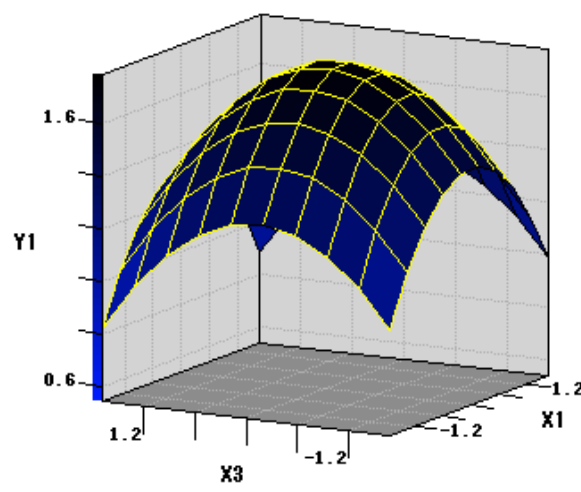

Fixed levels: $\quad \mathrm{x} 2=1.5 \mathrm{E}-6$

Figure 3. Contour plot and Response surface plot of lactose and fructooligosaccharides on the viable counts of BB01

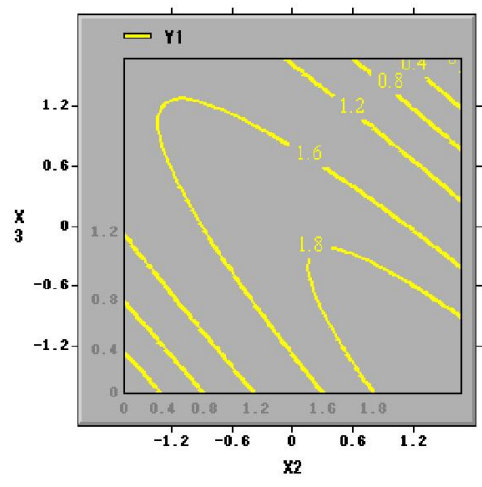

Fixed levels: $\quad \mathrm{H}_{1}=1.5 \mathrm{E}-\mathrm{B}$

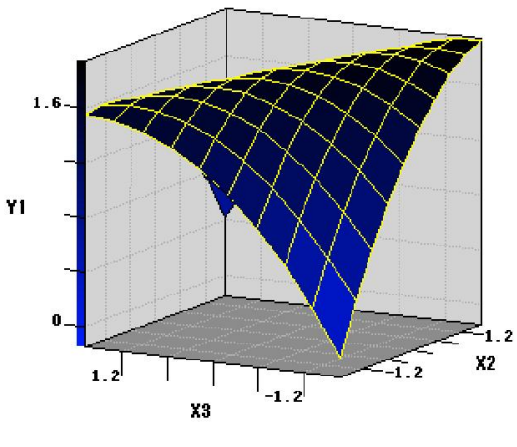

Fixed levels: $\quad \mathbf{x} 1=1.5 \mathrm{E}-\mathrm{6}$

Figure 4. Contour plot and Response surface plot of inulin and fructooligosaccharides on the viable counts of BB01

Response surface plot is very useful to determine the response value and the interactions between different factors. The round contour lines represent the weak interaction between variables, while the oval one implies the crucial interaction of variables. Figure 2 indicates the effect of the X1, X2 on the response value Y1. It can be suggested that the two-dimensional contour plots of them was almost round, which means the mutual interactions of them was not significant.

Figure 3 shows the response surface plot of effect of X1 and X3 on Y1. Similar to figure 2, the contour line seemed to be several circles, which represent the weak interaction between variables. Meanwhile, Figure 4 illustrates the effect of $\mathrm{X} 2, \mathrm{X} 3$ on the response value, we can easily see the contour line are oval, therefore, the significant interaction was clearly illustrated. 
Regression analysis was carried out using SAS software, worked out the partial derivatives of $\mathrm{X} 1, \mathrm{X} 2, \mathrm{X} 3$, then obtained the maximum value $(-0.174$, $1.123,-1.158)$. And converted the coded level into actual value: lactose, $1.6 \%$, inulin, $0.26 \%$ and fructooligosaccharides, $0.22 \%$. The predicted value of viable counts of Bifidobacterium bifidum under this condition was $2.15 \times 10^{9} \mathrm{cfu} / \mathrm{ml}$.

Validation of the model: The confirmatory experiments were conducted to test and verify the optimized result. Choosing MRS (lactose) culture medium as the control group $\left(\mathrm{M}_{0}\right)$, inoculum size was $5 \%$, then incubated the culture medium at $37 \square$ for $24 \mathrm{~h}$, and determined the OD value, $\mathrm{pH}$ value and the viable counts of BB01 at $12 \mathrm{~h}, 15 \mathrm{~h}, 18 \mathrm{~h}, 21 \mathrm{~h}$ respectively. The mean value of three parallel experiments was regarded as the final value, the result was shown in Fig.5, among then, the culture medium optimized by CCD was represented as M1.

It is assumed that the growth of BB01 shows an obvious increment in the optimized culture medium compared to the previous medium (Fig.5). When the incubation time was $18 \mathrm{~h}$, the OD value reached 1.434 , increased $6.58 \%$ compared to the control group. While the $\mathrm{pH}$ value showed a little decrease, which may because the acid producing ability of $\mathrm{BB}$ enhanced due to the increment of the carbon source. Meanwhile the viable counts of BB reached $(2.17 \pm 0.06) \times 10^{9} \mathrm{cfu} / \mathrm{mL}$, which increased $24.36 \%$ compared to the control group. This value is in the range of the predict value, this result proved that using RSM to optimize the compound carbon source and prebiotics of the culture medium is workable and can be accepted.

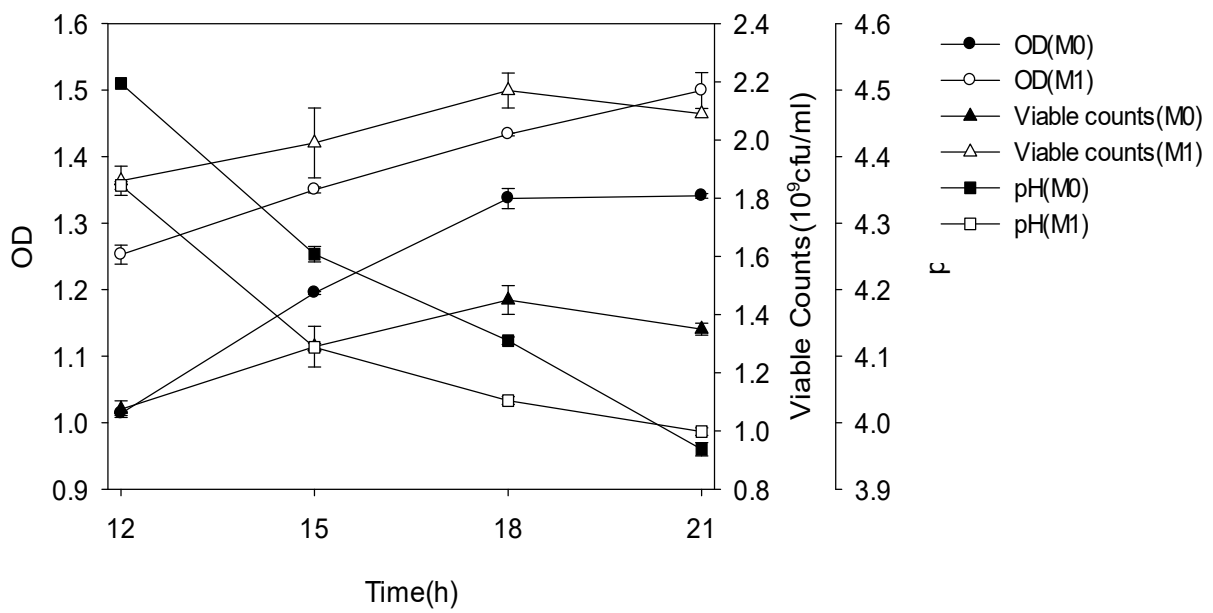

Figure 5. The verification test on Central Composite Design of carbon and prebiotics of $\mathrm{BB} 01\left(\mathrm{M}_{0}\right.$ : medium before optimization, $\mathrm{M}_{1}$ : medium after optimization) 


\section{DISCUSSIONS}

B. bifidum BB01 is a probiotics, which has been used in many foods, such as cheese and yogurt. The prebiotics has positive influence to the growth of $B$. bifidum $\mathrm{BB} 01$, which is because adding oligosaccharides (such as inulin and fructooligosaccharides) to the culture medium can promote the growth of probiotics. Oligosaccharides is a kind of prebiotics that are widely applied in yogurt. The prebiotics could be hydrolyzed by the enzyme in the probiotics, such as $\alpha$-gluosidase, $\beta$-glucosidase, $\alpha$-galactosidase and $\beta$-galactosidase. Hence, probiotics could hydrolyze and utilize the prebiotics to produce energy and promote the growth of it (Guo, 2006).

Carbon source is a fundamental energy producer for probiotics, B.A.Degnan (Hirokazu et al., 1995) indicates that Arabinogalactan could promote the growth of B. Longum. Ozer (Ozer et al., 2005) studied the effect of lactulose and inulin on the growth of L. acidophilus (LA-5) and B. Bifidum (BB02). The results presented that there is a positive influence between prebiotics and probiotics, especially lactulose. Inulin could stimulate the growth of $B$. Bifidum BB02 to a great extent. And lactulose was found to be more effective on the growth of both probiotic strains (LA-5 and BB02) than inulin. He et al. (2007) showed that different carbon source would affect the growth of Bifidobacterium bifidum, which is because that the expression of protein in probiotics was influenced by carbon source. Falony (Falony et al., 2009) found that different kinds of Bifidobacterium bifidum (B. breve Yakult, B. adolescentis LMG 10734 and B.angulatum LMG 11039) showed different metabolism on inulin, and fructooligosaccharides. These two oligosaccharides could promote the growth of three kinds of Bifidobacterium bifidum, which was related to the glycosidase produced eby $B$. Bifidum. The results of these studies indicate that carbon source and prebiotics had positive influence on the growth of probiotics, which was similar with our conclusion. Wasli (Wasli et al., 2009) conducted the medium optimization for Chitinase production from Trichoderma virens using center composite design, and indicated that with the optimized medium, the improvement of chitinase production was $80.97 \%$. It was proved that $\mathrm{CCD}$ is useful to optimize the medium. Torrades (Torrades et al., 2011) used CCD to optimize the degradation of black liquor. The results showed that the value of $\mathrm{UV}_{254}$ and $\mathrm{UV}_{280}$ were better under optimized conditions. This indicates that CCD was capable to optimize the degradation of black liquor. Zheng (Zheng et al., 2008) optimized the culture conditions for 1,3-propanediol by Klebsiella pneumoniae via central composite design. And the results showed that the 1,3-PD productivity of the batch culture increased $19.78 \%$ under optimized conditions. Our present study using CCD and RSM proved that CCD is 
qualified to optimize the amount of the carbon source and prebiotics for $B$. bifidum BB01.

\section{CONCLUSIONS}

Central composite design was employed to optimeze the carbon source and prebiotics (lactose, inulin and fructooligosaccharides) for B. bifidum BBO1 in this study. The selected carbon source and prebiotics had a significant effect on the viable counts of B. bifidum BBO1. The optimal concentrations of carbon source and prebiotics were lactose $1.6 \%$, inulin $0.26 \%$ and fructooligosaccharides $0.22 \%$, the the viable counts of $B$. bifidum BB01 increased $24.36 \%$ and reached $(2.17 \pm 0.06) \times 10^{9} \mathrm{cfu} / \mathrm{mL}$ compared to the control. Therefore, the CCD used in this study is workable for promoting the growth of B. bifidum BBO1.

\section{ACKNOWLEDGEMENTS}

The work was partly supported by the Science and Technology Overall Planning for innovation Engineering project of Shaanxi Province (No.2016KTCL02-30), Shaanxi Innovation and Transformation Project of Agricultural Science and Technology (No.NYKJ-2015-004) and project of academic leader team (No.2013XSD19).

\section{REFERENCES}

1. Baroutkoub A., Mehdi, R. Z., Beglarian R., et al. (2010). Effects of probiotic yogurt consumption on the serum cholesterol levels in hypercholestromic cases in Shiraz, Southern Iran. Sci. Res. Essays. 5 (16), 2206-2209.

2. Box, G. E. P. \& Wilson, K. B. (1951). On the experimental attainment of optimum conditions. J. Roy. Stat. Soc. (Ser. B) 13, 1-45.

3. De Vrese M., Steglman A., Richter B., et al. (2001). Probiotics-compensation for lactase insufficiency. Am. J. Clin. Nutr. 73, 421-429.

4. Falony G., Calmeyn T., Lero F, et al. (2009). Coculture fermentations of bifidobacterium species and bacteroides thetaiotaomicron reveal a mechanistic insight into the prebiotic effect of inulin-type fructans. Applied and Environmental Microbiology, 75(8), 2312-2319.

5. Goderska K. \& Czarnecki Z. (2007). Characterization of selected strains from Lactobacillus acidophilus and Bifidobacterium bifidum. Afr. J. Microbiol. Res. 1 (6), 065-078.

6. Guo X.F.. (2006). The study on the prebiotic effect of neoagarooligosaccharides. Master's dissertation, Ocean University of China, Shandong, China.

7. He T., Han R., Alvarez-Llamas G., et al. (2007). Differential analysis of protein expression of Bifidobacterium grown on different carbohydrates. $J$. Microbiol. Methods. 69(2), 364-370.

8. Hendrix, C. (1980). Through the response surface with test tube and pipe 
wrench. Chemtechol. 10, 488-497.

9. Holzapfel, W. H., Haberer P., Snel J., et al. (1998). Overview of gut flora and probiotics. Int. J. Food Microbiol. 41(2), 85-101.

10. Hirokazu O. \& Kenji Sonomoto. (1995). Arabinogalactan Utilization in Continuous Cultures of Bifidobacterium longum: Effect of Co-culture with Bacteroides thetaiotaomicron. Anaerobe. 1(2), 103-112.

11. Kabeerdoss J., Devi, R. S., Mary, R. R., et al. (2011). Effect of yoghurt containing Bifidobacterium lactis $\mathrm{Bb} 12$ on faecal excretion of secretory immunoglobulin A and human beta-defensin 2 in healthy adult volunteers. Nutr. J. 10(1), 1-4.

12. Kalil, S. J., Maugeri F, \& Rodrigues, M. I. (2000). Response surface analysis and simulation as a tool for bioprocess design and optimization. Proc. Biochem. 35(6), 539-550.

13. Mainville I., Arcand Y. \& Farnworth, E. R. (2005). A dynamic model that simulates the human upper gastrointestinal tract for the study of probiotics. Int. J. Food Microbiol. 99 (3), 287-296.

14. Ozer D., Akin S. \& Ozer B. (2005). Effect of inulin and lactulose on survival of lactobacillus acidophilus LA-5 and bifidobacterium bifidum BB-02 in acidophilus-bifidus Yoghurt. Food Science and Technology International. 11(1), 19-24.

15. Rafter, J. (2004). Probiotics and colon cancer. Nutrition research reviews. 17(2), 277-284.

16. Schiffrin, E. J. \& Blum S. (2001). Food processing: probiotic microorganisms for beneficial foods. Curr. Opin. Biotechnol. 12(5), 499-502.

17. Torrades F., Saiz S., Garcia-Hortal J.A. (2011). Using central composite experimental design to optimize the degradation of black liquor by Fenton reagent. Desalination. 268(1-3):97-102.

18. Veronique C., M. Feinberg, C. Constantin. \& D.Christian (1983). Application of response surface methodology to evaluation of bioconversion experimental conditions. Appl. Environ. Microbiol. 45(2), 634-639.

19. Wang S., Zhu H., Lu C., et al. (2012). Fermented milk supplemented with probiotics and prebiotics can effectively alter the intestinal microbiota and immunity of host animals. J. Dairy Sci. 95(9), 4813-4822.

20. Wasli A.S., Salleh M.M., Aziz S.A., et al. (2009). Medium optimization for chitinase production from Trichoderma virens using central composite design. Biotechnology \& Bioprocess Engineering. 14(6):781-787.

21. Zheng Z.M., Hu Q.L., Hao J., et al. (2008). Statistical optimization of culture conditions for 1,3-propanediol by Klebsiella pneumoniae AC 15 via central composite design. Bioresource Technology. 99(5):1052-1056. 